\title{
Multiple Myeloma: The Success Story Continues
}

\author{
Marcus Hentrich \\ Department of Medicine III, Hematology/Oncology, Red Cross Hospital Munich, Munich, Germany
}

Multiple myeloma (MM) is the second most common hematological malignancy worldwide with 7,600 estimated new cases in 2020 in Germany and 34,920 estimated new cases in 2021 in the USA [1,2]. During the last 2 decades, tremendous advances have been made in the understanding of myeloma biology and the development of improved treatment strategies $[3,4]$. The availability of highly effective novel drugs such as proteasome inhibitors, immunomodulatory drugs, and monoclonal antibodies targeting CD38 or signaling lymphocytic activation molecule family member 7 (SLAMF7) have led not only to higher response rates but also to improved progression-free survival and overall survival rates. Even 25 years after publication of the results of the first randomized trial on melphalan-based high-dose chemotherapy and autologous stem cell transplantation, this intensified treatment approach remains the treatment of choice for eligible patients with newly diagnosed MM [4-6]. As undetectable minimal residual disease (MRD) has been shown to be a valuable surrogate for progression-free survival and overall survival, MRD testing is now an integral part of current clinical trials [7, 8]. Further, the advent of B-cell maturation antigen-targeted therapies such as antibody-drug conjugates, bispecific antibody constructs, and in particular chimeric antigen receptor-modified $\mathrm{T}$ cell therapies represents a promising step forward.

The objective of this review series was to provide an update on recent developments in the understanding of the molecular pathogenesis and disease evolution of MM and to give an overview on current treatment approaches in newly diagnosed and relapsed and refractory MM.

In the first article of the present focus, Heider et al. [9] summarize current knowledge of the pathogenesis of $\mathrm{MM}$, which is crucial for the development of novel tar- geted therapeutic approaches. The authors show very clearly that primary genetic lesions such as translocations and hyperdiploidy contribute to pathogenesis as well as somatic mutations in various oncogeneic pathways, epigenetic alterations, and a tumor-promoting bone marrow microenvironment. Gandili et al. [10] review current treatment approaches to newly diagnosed MM emphasizing the goal of inducing a rapid and deep remission which ideally should lead to undetectable MRD. The article by Knop and Heimberg [11] gives an up-to-date overview on the management of relapsed and refractory MM. The authors point to the promising efficacy and safety of triplet combinations containing anti-CD38 monoclonal antibodies in combination with next-generation compounds. Finally, in their article on long-term treatment of MM in a routine care outpatient clinical setting, Steinmetz et al. [12] report that $44 \%$ and $30 \%$ of patients were estimated to reach 4th and 5th line of therapy, respectively. These numbers are markedly higher than those reported in cross-sectional studies indicating a broad spectrum of drugs available and that later lines of therapy should be brought more into focus.

\section{Conflict of Interest Statement}

The author served on advisory boards of Amgen, EusaPharma, Janssen and Takeda, and received lecture fees by Amgen, Celgene, Janssen, Sanofi and Takeda.

\section{Funding Sources}

This research did not receive any specific grant from funding agencies in the public, commercial, or non-for-profit sectors. 


\section{References}

1 Zentrum für Krebsregisterdaten. Krebs in Deutschland für 2015/2016. 12. Ausgabe. Korrigierte Fassung vom; 2020. Available from: https://www.krebsdaten.de/Krebs/DE/ Content/Publikationen/Krebs_in_Deutschland/kid_2019/krebs_in_deutschland_2019. pdf?_blob=publicationFile.

2 Siegel RL, Miller KD, Fuchs HE, Jemal A. Cancer Statistics, 2021. CA Cancer J Clin. 2021;71(1):7-33.

3 Rodriguez-Otero P, Paiva B, San-Miguel JF. Roadmap to cure multiple myeloma. Cancer Treat Rev. 2021;100:1002284

4 Dimopoulos MA, Moreau P, Terpos E, Mateos MV, Zweegman S, Cook G, et al. Multiple myeloma: EHA-ESMO clinical practice guidelines for diagnosis, treatment and follow-up. Ann Oncol. 2021;32(3):309-22.

5 Attal M, Harousseau J-L, Stoppa A-M, Sotto JJ, Fuzibet JG, Rossi JF, et al. A prospective, randomized trial of autologous bone marrow transplantation and chemotherapy in multiple myeloma. N Engl J Med. 1996;335:91-7.

6 Gay F, Mina R, Rota-Scalabrini D, Galli M, Belotti A, Zamagni E, et al. Carfilzomib-based induction/consolidation with or without autologous transplant (ASCT) followed by lenalidomide $(\mathrm{R})$ or carfilzomib-lenalidomide (KR) maintenance: efficacy in high-risk patients. J Clin Oncol. 2021;39(1):8002.

7 Munshi NC, Avet-Loiseau H, Anderson KC, Neri P, Paiva B, Samur M, et al. A large metaanalysis establishes the role of MRD negativity in long-term survival outcomes in patients with multiple myeloma. Blood Adv. 2020;4: 5988-999.

8 Cavo M, San-Miguel JFF, Usmani SZ, Weisel KC, Dimopoulos MAA, Avet-Loiseau H, et al. Prognostic value of minimal residual disease negativity in myeloma: combined analysis of
POLLUX, CASTOR, ALCYONE, MAIA. Blood. 2021;Jul 21:blood.2021011101.

9 Heider M, Nickel K, Högner M, Bassermann F. Multiple myeloma: molecular pathogenesis and disease evolution. Oncol Res Treat. 2021. DOI: $10.1159 / 000520312$.

10 Ghandili S, Weisel KC, Bokemeyer C, Leypol$\mathrm{dt}$ LB. Current treatment approaches to newly diagnosed multiple myeloma. Oncol Res Treat. 2021. DOI: 10.1159/000520504.

11 Heimberg L, Knop S. Updated perspectives on the management of relapsed and refractory multiple myeloma. Oncol Res Treat. 2021. DOI: $10.1159 / 000520364$

12 Steinmetz T, Ernst A, Hellmich M, Heinz M, Totzke U. Effectiveness of long-term treatment of multiple myeloma in regular care comparison of a longitudinal and a cross-sectional analysis approach. Oncol Res Treat. 2021. DOI: 10.1159/000519419. 
